\title{
Dynamique urbaine à Prague de la fin de la période communiste à nos jours : l'importation du modèle occidental du commerce de détail
}

Urban dynamic in Prague from the end of the communist period to the present day: the import of western retail organization

Städtische Dynamik in Prag vom Ende der kommunistischen Periode bis heute : die Einführung des westlichen Modells der Einzelhandelsorganisation

\section{Carole Pommois}

\section{OpenEdition}

Journals

Édition électronique

URL : http://journals.openedition.org/rge/4418

DOI : $10.4000 /$ rge.4418

ISSN : 2108-6478

Éditeur

Association des géographes de l'Est

Édition imprimée

Date de publication : 1 avril 1999

ISSN : 0035-3213

\section{Référence électronique}

Carole Pommois, « Dynamique urbaine à Prague de la fin de la période communiste à nos jours

l'importation du modèle occidental du commerce de détail », Revue Géographique de l'Est [En ligne], vol. 39 / 2-3 | 1999, mis en ligne le 05 septembre 2013, consulté le 08 septembre 2020. URL : http:// journals.openedition.org/rge/4418; DOI : https://doi.org/10.4000/rge.4418

Ce document a été généré automatiquement le 8 septembre 2020

Tous droits réservés 


\title{
Dynamique urbaine à Prague de la fin de la période communiste à nos jours : l'importation du modèle occidental du commerce de détail
}

\author{
Urban dynamic in Prague from the end of the communist period to the present \\ day: the import of western retail organization \\ Städtische Dynamik in Prag vom Ende der kommunistischen Periode bis heute : \\ die Einführung des westlichen Modells der Einzelhandelsorganisation
}

\section{Carole Pommois}

1 Si les villes occidentales sont relativement bien connues des géographes français, il n'en est pas de même pour les villes d'Europe Centrale et Orientale (PECO). Durant une quarantaine d'années, ces villes ont suivi une évolution bien différente de celle des villes ouest-européennes. Les mécanismes de marché disparurent dans le cadre d'une économie dite de commandement. L'essentiel du bâti était nationalisé. La croissance des villes, notamment celle des capitales, était contrôlée. Les espaces urbains faisaient l'objet d'une planification répondant à la volonté des régimes communistes d'homogénéiser l'espace.

En 1989, on vit tomber les régimes communistes est-européens. Des régimes de transition furent mis en place afin de faciliter le passage d'une économie de commandement à une économie fonctionnant à nouveau selon les règles du marché. À l'instar des changements politiques, économiques, sociaux et culturels, les espaces urbains subissent eux aussi les effets de la transition. L'analyse de la mutation des villes des PECO dans le nouveau contexte des années 1990 revient à nous interroger sur les résultats de la politique socialiste sur l'organisation spatiale des villes et sur la transition en tant que passage d'une organisation spatiale à une autre. Ces questions sont abordées par l'analyse d'un cas particulier : l'organisation commerciale de Prague, capitale de la République Tchèque. 


\section{La transition a commencé à la fin du régime communiste}

3 Depuis l'instauration du régime communiste, la production des biens de consommation fut quelque peu négligée au profit du secteur des industries lourde, chimique et électronique. De manière générale, les biens produits étaient de mauvaise qualité et peu diversifiés. En 1986, même les produits des industries traditionnelles tchèques étaient touchés. Cette année fut effectivement qualifiée d'année noire pour l'industrie légère. Quant au secteur de la petite distribution, les directives de vente étaient décidées au niveau central à Prague. Les prix étaient fixés par le ministère du Commerce intérieur et du Tourisme et étaient les mêmes dans tout le réseau de commerces de détail. Les besoins de la population étaient calculés selon des normes strictes. Face au constat de l'échec des précédents plans quinquennaux et sous l'influence de la politique menée par M. Gorbatchev en Union Soviétique, les autorités tchécoslovaques réagirent en annonçant une série de réformes structurelles des entreprises dans le cadre du 8e plan quinquennal 1986-1990.

\section{A. Le huitième plan quinquennal, une tentative de transition économique}

\section{- Le secteur de la production}

4 Le 8e plan prévoyait la construction de nouvelles usines et la modernisation de certaines branches de l'industrie légère, notamment celles des bijoux de fantaisie, de la porcelaine, du verre et du cuir. L'accent était principalement mis sur la qualité des produits et l'efficacité de la production. Mais de nombreuses entreprises continuèrent à fonctionner comme au cours des précédents plans quinquennaux, en produisant les quantités prévues au détriment de la qualité.

5 Les réformes concernaient également la gestion des industries. Tout en ne remettant pas en cause le principe du contrôle central, les autorités tchécoslovaques semblaient prêtes à accorder une certaine autonomie aux entreprises. Certaines d'entre elles avaient même le droit de commercer avec l'Ouest. M. Gorbatchev avait réussi, lors de sa visite en Tchécoslovaquie du 9 au 12 avril 1987, à convaincre les dirigeants de mettre en route le nouveau mode de gestion des entreprises ${ }^{1}$. Il s'agissait de mettre en place des réformes alors que le printemps de Prague de 1968 était encore bien présent dans les esprits.

\section{- Le secteur de la distribution}

Le 8e plan quinquennal prévoyait également pour ce secteur l'injection de mécanismes nouveaux, inspirés de ceux de l'économie de marché: autonomie des entreprises, compétition, flexibilité des prix, profits, mais, là aussi, sans toucher au contrôle central (Summary of world broadcast, 18/07/87).

7 Dès le début des années 1950, la part socialisée du commerce de détail était largement supérieure à $80 \%$ en Tchécoslovaquie. Trois systèmes de propriétés coexistaient: les entreprises d'État, les entreprises privées et les coopératives. Les entreprises privées 
devaient disparaitre progressivement. Les coopératives étaient, quant à elles, étroitement surveillées par les entreprises d'État.

8 Ces grandes entreprises publiques étaient organisées par branche d'activité. Chacune portait le nom de l'assortiment dont elle avait en charge la distribution et détenait le monopole de son secteur. Elles étaient directement subordonnées au ministère du Commerce intérieur et du Tourisme. En 1968, une loi fédérale scinda ces entreprises d'État en deux branches, l'une tchèque et l'autre slovaque. Mais les directives de vente continuèrent à être définies au niveau central, à Prague. Ce n'est qu'en 1988, dans le cadre de la Perestroïka, que l'on vit apparaître un échelon supplémentaire: les directions régionales.

9 La construction de nouvelles surfaces commerciales (petites unités, supermarchés et centres commerciaux dans les cités résidentielles) fut également entreprise au cours de l'année 1986. Néanmoins, le bilan ne fut guère plus positif que celui relatif au secteur de la production des biens de consommation courante. Les autorités tchécoslovaques durent constater l'inadaptation du réseau de commerce, la faiblesse des investissements dans ce secteur et le manque de personnel. Le nombre de petits commerces continua à baisser jusqu'en 1989 (tableau 1).

Tableau 1 : Évolution du commerce de détail à Prague vers la fin du régime communiste

\begin{tabular}{|l|c|c|c|c|c|c|c|c|c|c|c|c|}
\hline Années & 1970 & 1975 & 1980 & 1981 & 1982 & 1983 & 1984 & 1985 & 1986 & 1987 & 1988 & 1989 \\
\hline $\begin{array}{l}\text { Nombre } \\
\text { d'établissements }\end{array}$ & 4519 & 4661 & 4158 & 4042 & 4003 & 3956 & 3925 & 3905 & 3886 & 3874 & 3860 & 3821 \\
\hline
\end{tabular}

\section{- L'échec des tentatives de réformes}

Malgré la volonté de réforme des autorités tchécoslovaques, le marché demeura incapable de répondre aux besoins sans cesse croissants de la population. Celle-ci se tourna alors vers le marché noir qui prit une ampleur internationale à partir de 1988. De véritables réseaux de commerces parallèles se mirent en place, non seulement dans les zones frontalières, mais également dans les villes de l'intérieur. Les Tchèques et les Slovaques pratiquaient le tourisme de shopping dans les autres pays du Conseil d'Assistance Économique Mutuelle (CAEM). La Tchécoslovaquie réagit d'ailleurs vivement contre ce phénomène, invoquant la protection de son commerce intérieur (J. Blaha, 1989).

11 À Prague, les habitants pouvaient s'approvisionner auprès des vendeurs de rue, dans les kiosques, les marchés de fruits et légumes et les marchés asiatiques. On vit même vers la fin de la période communiste une certaine légalisation de ces formes de commerce.

\section{B. Le maintien du commerce de détail dans le centre-ville}

\section{- L'État décidait de la localisation du commerce de détail}

12 Les communistes avaient élaboré un modèle de la ville socialiste selon lequel les services, de qualité égale, devaient être accessibles à tous les citadins. L'espace urbain faisait l'objet d'une planification dans le cadre d'un schéma directeur, adopté pour une période de dix ans et prévoyant les grandes orientations. À Prague, le dernier schéma directeur fut adopté en 1986. 
13 De la même façon que les besoins de la population étaient calculés selon des normes strictes, la localisation du commerce de détail était décidée par les autorités de la ville, selon des critères qui peuvent rappeler les mécanismes de marché : accessibilité, caractéristiques des commerces existants, clientèle. Un certain nombre de commerces étaient ainsi prévus par aire géographique.

\section{- La centralité du centre-ville fut maintenue}

Comme pour toutes les villes des pays est-européens, la centralité commerciale du centre-ville fut largement privilégiée. Prague ne connut pas la naissance des grands centres commerciaux qui caractérisent si bien les périphéries des villes occidentales et leur centre-ville. L'appareil commercial praguois était composé en quasi majorité de petites unités de commerce. Il existait cependant des chaînes de supermarchés alimentaires.

L'égalité relative des valeurs locatives pratiquées à Prague permit le maintien d'activités commerciales peu rentables et l'installation d'entrepôts dans le centre-ville. À la fin de la période communiste, ce dernier était également caractérisé par un grand nombre de locaux non-utilisés.

La centralité commerciale du centre-ville était d'autant plus forte que plus de la moitié de la population vivait dans les grandes cités-dortoirs, bâties comme une réponse à la pénurie de logements, à partir de la fin des années 1950. Ces grands ensembles constituaient de véritables déserts commerciaux. Pour y remédier, des centres de quartiers comprenant une grande surface alimentaire et quelques commerces, en général tous bâtis selon le même modèle, furent construits. Mais les produits et les services proposés restaient largement insuffisants. Pour les achats spécifiques, comme pour les achats quotidiens, les Praguois devaient se rendre dans le centre-ville.

17 La transition amorcée avant 1989 concerne d'abord les structures et les modes de gestion des entreprises de production et de distribution. Concernant l'organisation spatiale du commerce de détail, il faudra attendre la privatisation pour voir s'opérer des changements profonds.

\section{La mise en place d'une nouvelle organisation commerciale dans le cadre de la transition}

18 L'échec des diverses tentatives de réforme menées durant la fin des années 1980 conduisirent à la chute du Mur de Berlin et des régimes communistes est-européens. À partir de 1991, la Tchécoslovaquie ${ }^{2}$ engagea des processus de restitution, de privatisation et de libéralisation. Une fois ces processus amorcés, le paysage commercial de la ville commença à se modifier.

19 Entre 1989 et 1991, il y eut une première période de transition qui se déroula dans la continuité des réformes des années précédentes. En 1990, on vit apparaître un second échelon dans l'organisation des grandes entreprises d'État: les directions de district. Il s'agissait avant tout d'un processus de décentralisation. La véritable mutation du commerce de détail ne débuta qu'à partir de 1991 lorsque les premiers petits commerces furent transférés au secteur privé par le biais de la restitution et de la petite privatisation ${ }^{3}$. 


\section{A. Les effets de la transition sur le commerce de détail}

\section{- Une fragmentation de l'appareil commercial tchèque et une augmentation du nombre d'indépendants}

Dès 1990, la liberté des affaires privées fut introduite par la loi relative aux entreprises privées. Cette loi fut complétée en 1992 par un autre texte, relatif aux licences commerciales (Zivnostensky zakon en tchèque). Par une simple inscription sur un registre de commerce, les Tchèques et les Slovaques purent s'essayer dans les affaires. Beaucoup échouèrent, cessèrent d'exercer leur activité commerciale et oublièrent de le signaler. D'autres, profitant du nouveau contexte de liberté, changèrent d'activité. Jusqu'à aujourd'hui, le commerce de détail se caractérise par sa très courte durée de vie et par le changement rapide de fonction. Cette loi s'applique également à toute personne étrangère, physique ou morale, désirant exercer une activité commerciale en République Tchèque.

21 Le commerce de détail fut privatisé par le biais de trois processus que sont la restitution, la petite privatisation et la grande privatisation. L'un des premiers effets de la restitution et de la petite privatisation a été la fragmentation de l'appareil commercial tchèque. La plupart des grandes entreprises publiques disparurent, la privatisation s'étant effectuée au niveau de l'établissement commercial. Celles qui survécurent durent se spécialiser. En conséquence directe, le nombre d'indépendants augmenta. Tout citoyen tchèque et slovaque majeur pouvait participer à ce processus, tandis que la participation étrangère fut interdite lors de la première phase du processus de petite privatisation.

Dans un premier temps, l'augmentation du nombre de commerces s'est effectuée par la restauration des anciens locaux commerciaux dans le centre-ville et les quartiers anciens de Prague, la reprise des centres de quartier des cités-dortoirs et l'occupation des parties communes, situées dans les rez-de-chaussée des immeubles de ces mêmes cités ${ }^{4}$. Un autre phénomène qui peut être observé, est celui de la conversion des anciens sites industriels en espaces de bureau et de commerce.

\section{- L'internationalisation du commerce de détail}

En janvier 1991, le commerce extérieur fut totalement libéralisé. L'économie tchèque se réorienta dès lors vers le marché occidental. Un autre processus important de l'internationalisation du commerce de détail est celui de la restitution des immeubles nationalisés après le Coup de Prague du 25 février 1948. Cette restitution permit la réémergence d'un marché immobilier et foncier ouvert aux investisseurs et sociétés étrangères. Lorsque la participation étrangère fut enfin permise dans le processus de privatisation, les sociétés internationales purent investir à Prague. L'ensemble de ces mécanismes allait conduire à une internationalisation rapide du commerce de détail. nouveaux propriétaires : la faiblesse des loyers, maintenue délibérément dans le secteur résidentiel en vue de la protection des locataires $^{5}$, incite les nouveaux propriétaires à transformer des appartements en espaces de bureau et de commerce. Une société étrangère ou internationale, ayant constitué une société de droit tchèque, peut alors se porter acquéreur. 

investisseurs occidentaux : des promoteurs occidentaux investissent ainsi dans l'immobilier et entreprennent des travaux de réhabilitation. Le résultat est la transformation de l'immeuble en centre de bureaux et de commerces. En 1994, les premiers centres commerciaux apparaissent dans et à proximité du centre-ville. Les promoteurs occidentaux lancent des opérations immobilières ou foncières, non seulement dans les vieux quartiers de Prague, mais aussi à sa périphérie, à proximité des autoroutes et des grandes cités-dortoirs. Le premier centre commercial «Cerny Most Centrum ", comprenant un supermarché (environ $2000 \mathrm{~m} 2$ ), un magasin Marks\&Spencer, un établissement Mac Donald's et des commerces de luxe, a ouvert ses portes tout à la fin de l'année 1997, à proximité immédiate d'une cité-dortoir (Fig. 2).

Les grandes chaînes de distribution étrangères se font de plus en plus présentes dans le paysage commercial. Quelques chaînes de distribution nationales (Delvita pour l'alimentation) se développent également. Leur part sur le marché tchèque reste cependant limité par rapport à ce qui est connu en Occident.

- Les entreprises internationales s'implantent en premier lieu dans le centreville : la présence étrangère ne se réduit pas à ces centres commerciaux et supermarchés. Les grandes marques internationales ouvrent des points de vente dans la capitale tchèque. Un phénomène illustrant l'occidentalisation du paysage urbain est l'implantation massive de la chaîne de restauration rapide Mac Donald's à travers la capitale et ses environs. Peu après, une autre chaîne américaine, Kentucky Fried Chicken, ouvrit ses premiers établissements dans le centre-ville. Certains, à Prague, mécontents de cette présence américaine, parlent de Mac Donaldisation de la ville (J. Kaplan et K. Nosarzewska, 1997). Les autres grandes enseignes internationales ne sont pas absentes du paysage. On trouve, par exemple, un établissement United Colors of Benetton à côté d'une enseigne Adidas. Les grands cosmétiques français sont également bien représentés.

28 À côté de ces marques de renommée internationale, des particuliers étrangers viennent s'installer à Prague, saisir une occasion de réussir dans les affaires.

\section{- Le développement des autres formes de commerce}

29 À côté des établissements classiques et des centres commerciaux, d'autres formes de commerces prennent une nouvelle ampleur : il s'agit notamment des marchés de fruits et légumes, de plus en plus présents à travers la capitale. L'assortiment proposé n'est pas très large. L'essentiel consiste en pommes, bananes, oranges, choux, poivrons, tomates. Les marchés vestimentaires asiatiques sont également bien présents dans le paysage de Prague. Souvent, on les trouve auprès des marchés de fruits et légumes. On peut également trouver un stand isolé, dans les centres de quartier des cités-dortoirs par exemple.

Un autre forme de commerce qui avait son importance avant 1989 est celle des kiosques où l'on peut trouver un large assortiment, allant de l'alimentaire au vestimentaire, de la droguerie à la parfumerie. Ces kiosques sont en général localisés à la sortie du métro et aux arrêts de tramway, particulièrement dans les quartiers périphériques. Ils ont tendance à disparaître dans le centre-ville. Par contre, les kiosques à journaux, les kiosques obcerstveni (rafraîchissement) où l'on peut s'acheter un hot dog ou une boisson, et ceux où l'on peut se procurer des tickets pour des visites guidées de la ville, 
sont nombreux au cœur même du quartier des affaires de Prague, également haut lieu du tourisme international. Les kiosques fleuristes sont aussi bien représentés.

Enfin, le troisième phénomène est celui des vendeurs de rue ou marchands ambulants. Dans le centre-ville, ces vendeurs jalonnent les rues touristiques. Leur assortiment est peu varié. D'un bout à l'autre du circuit touristique traditionnel, on trouve les mêmes articles, principalement des bijoux fantaisie, des lunettes de soleil, divers petits bibelots. Certains ont investi des façades commerciales abandonnées, les porches ou les entrées des immeubles. La plupart du temps, la marchandise est posée. La vente ambulante en voiture est également une pratique courante.

D'autres formes de commerce, entièrement nouvelles pour le pays, apparaissent. Il s'agit notamment de la vente par correspondance et du télé achat ou télé shopping.

\section{- Le phénomène de sélection des activités commerciales dans le centre-ville}

Un autre processus important de la mutation du paysage commercial de la ville de Prague est celui de la libéralisation des valeurs locatives dans les secteurs non résidentiels. Cette libéralisation s'est effectuée à divers degrés selon la localisation dans la ville. Totale dans le centre-ville et les quartiers environnants, elle n'est que partielle dans d'autres secteurs. Certaines communes (en général rurales) ${ }^{6}$ bénéficient de l'autorité pour décider d'un plafond pour les valeurs locatives même pour les surfaces de bureau et de commerce. On assiste à un phénomène de sélection qui n'existait pas dans le centre-ville sous l'ancien régime. Les commerces de luxe remplacent progressivement les commerces de quartier. Les commerces et les services à caractère commercial se tournent de plus en plus vers une clientèle touristique internationale.

En 1991, une loi obligea les nouveaux propriétaires à conserver, pour une durée d'une année au moins, le commerce alimentaire qui se trouvait dans leur immeuble. Le principal objectif de ce nouveau texte était le maintien des commerces de quartier dans le centre-ville où les résidants sont encore particulièrement nombreux. Mais, à Prague, où la législation est encore à établir, cette loi n'eut pas d'impact considérable et ne put empêcher la baisse progressive du nombre de ces petits commerces alimentaires. De plus, ces derniers subissent la concurrence des grandes surfaces alimentaires tchèques et occidentales.

Le quartier des affaires du centre-ville se caractérise aujourd'hui par ses vitrines prestigieuses. En dehors du cœur de la capitale et de son itinéraire touristique, les vitrines n'attirent pas particulièrement l'œil du visiteur occidental.

\section{B. Les effets de la transition sur l'organisation commerciale}

La transition n'a pas que des conséquences sur les formes de commerce et les structures. L'organisation du commerce de détail et des services à caractère commercial est également concernée. On peut distinguer trois phases dans son évolution actuelle.

\section{- Les deux premières phases : le centre-ville demeure le premier centre commerçant et commercial}

Dans un premier temps, le développement a essentiellement consisté en la multiplication des commerces indépendants. Jusqu'à l'apparition des premiers centres 
commerciaux en 1994, il n'y a quasiment pas eu construction de nouvelles surfaces commerciales. S'agissant d'une reprise de l'ancien réseau de commerces, le centre-ville s'est vu conforté dans sa place de premier centre commerçant. Les nouveaux établissements commerciaux, apparus dans les cités-dortoirs, ne suffisent pas à concurrencer le centre-ville. Encore aujourd'hui, le centre de Prague se caractérise par une densité commerciale inégalée des autres quartiers de la ville. Les commerces ouvrent dans les cours, les étages ou les sous-sols des immeubles.

Une deuxième phase de l'évolution commerciale de la capitale tchèque commence en 1994 avec l'ouverture des premiers centres commerciaux dans le centre-ville et les quartiers voisins. Ce phénomène est tardif par rapport au processus de restitution en raison des délais, extrêmement longs, des opérations immobilières et de l'obtention des autorisations nécessaires auprès des autorités de la ville. Ce développement se poursuit encore de nos jours, même dans le centre-ville, bien que la densité du bâti ne permette que l'implantation de petites unités de commerces (Photo 1).

Photo 1 : Deux grands magasins spécialisés sur la place Wenceslas : les chaussures Bata qui retrouvent leur patrie d'origine et une toute nouvelle librairie, ouverte en 1998

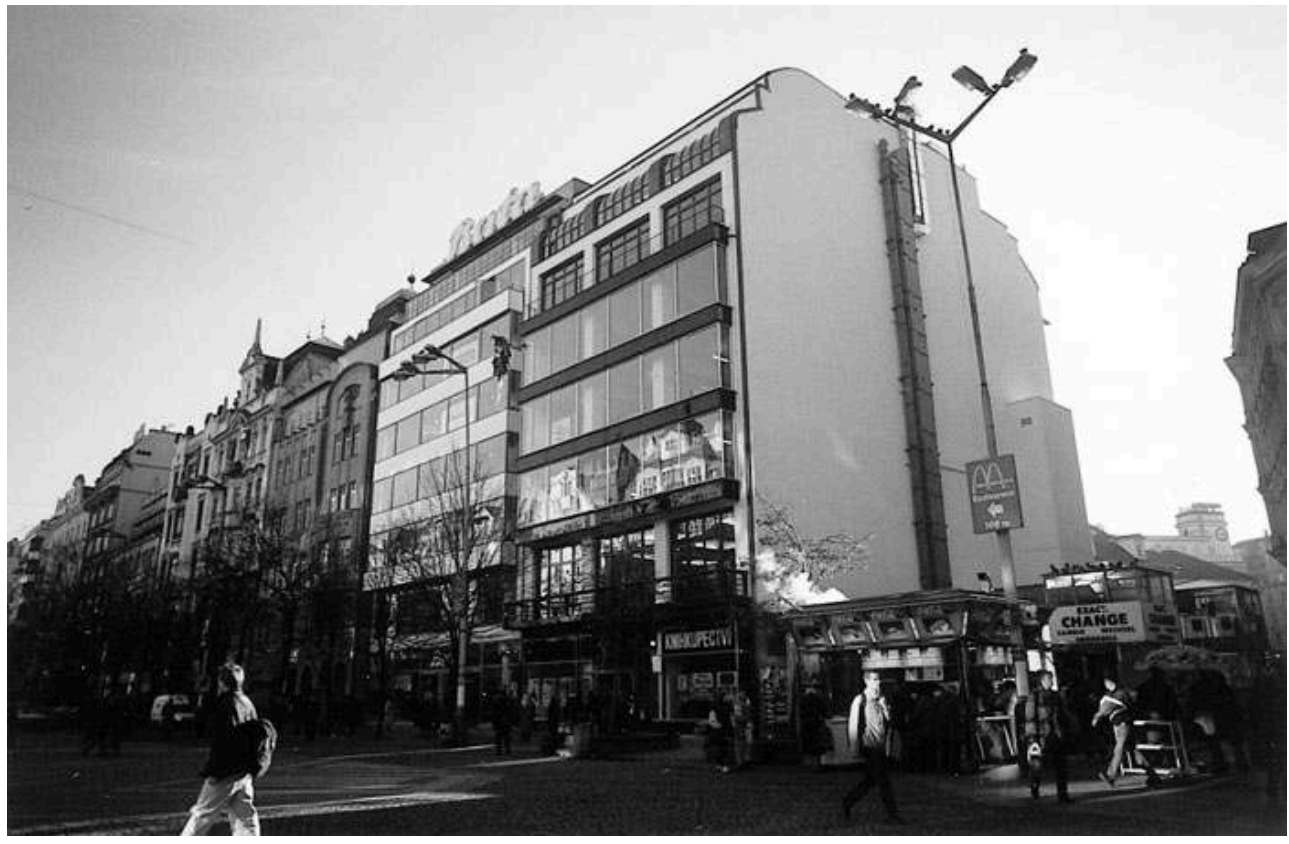

Cliché : Carole Pommois.

Au plein cœur du centre-ville, l'ouverture des nouveaux centres commerciaux et des grands magasins nécessite d'importants travaux de restauration. Sous le régime communiste, le bâti du centre-ville fut le sujet d'une dégradation progressive en raison de la politique urbaine qui privilégie la construction de nouveaux ensembles résidentiels à l'entretien des immeubles anciens.

39 La figure 1 représente un historique des processus qui se sont mis en place à partir de 1989 et qui aboutissent, jusqu'à aujourd'hui, au maintien de la centralité commerciale du centre-ville. 
Figure 1 : Schéma des processus de la mutation du commerce de détail à Prague à partir de 1989

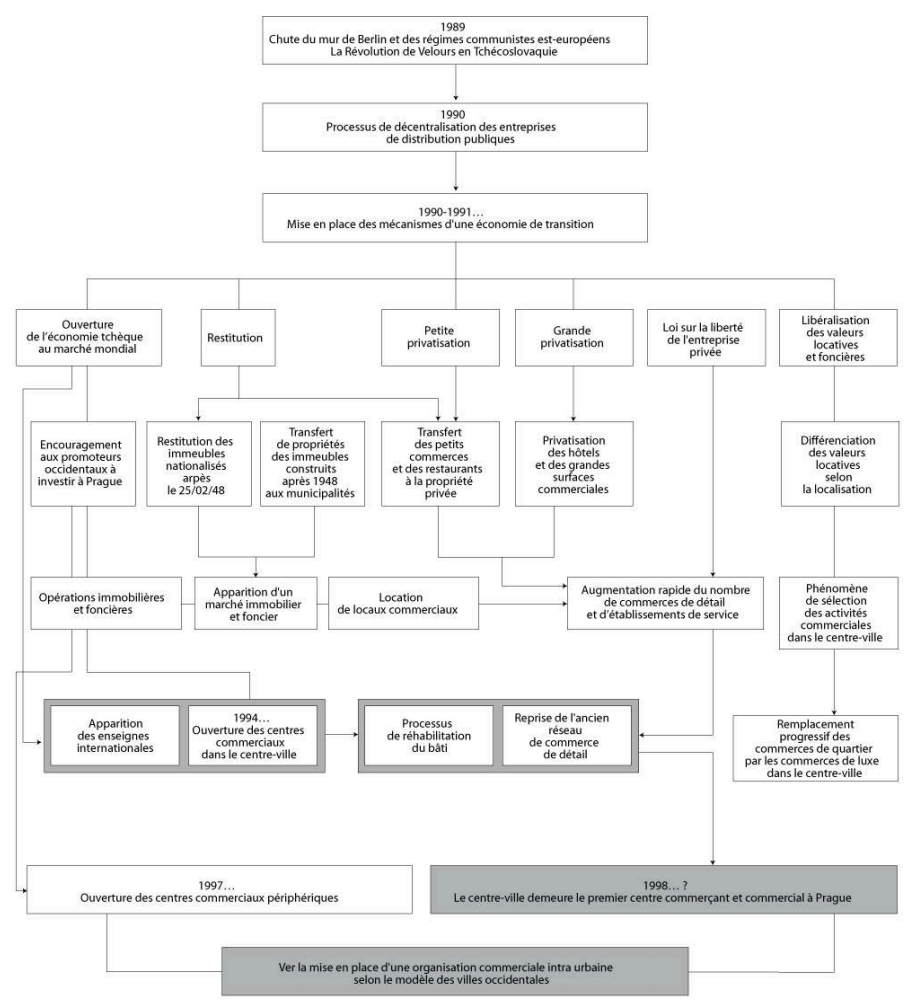

Sources : Foucher M. 1993. Haeley \& Baker. 1998.

- La troisième phase : une accélération de la mutation commerciale de Prague ; l'ouverture des centres commerciaux périphériques (fig. 2)

Ces deux phénomènes, la multiplication des petits établissements commerciaux et l'apparition des centres commerciaux dans les vieux quartiers de Prague, se font de façon spontanée. Ce n'est que dans une troisième phase du développement du commerce de détail que les autorités de la ville proposent la création de centres commerciaux périphériques à l'image de ceux des villes occidentales. Ce développement est également tardif en raison de la durée des négociations avec les municipalités concernées. Ces centres entrent dans le cadre d'une politique de protection du centre-ville, classé patrimoine historique par l'Unesco. Le principal souci de la ville est un désengorgement du centre où les conditions de circulation deviennent exécrables avec l'augmentation du parc automobile tchèque. Les nouveaux centres seront tous localisés à proximité des voies rapides et des cités-dortoirs, dont certaines sont encore en cours de construction. A priori, cela ne devrait pas entraîner une baisse de dynamisme pour le centre-ville en raison du tourisme international de masse. Jusqu'à présent, le centre-ville demeure le premier centre commerçant et commercial de Prague. 
Figure 2 : L'ouverture des centres commerciaux périphériques à Prague. Carte de localisation, 1998

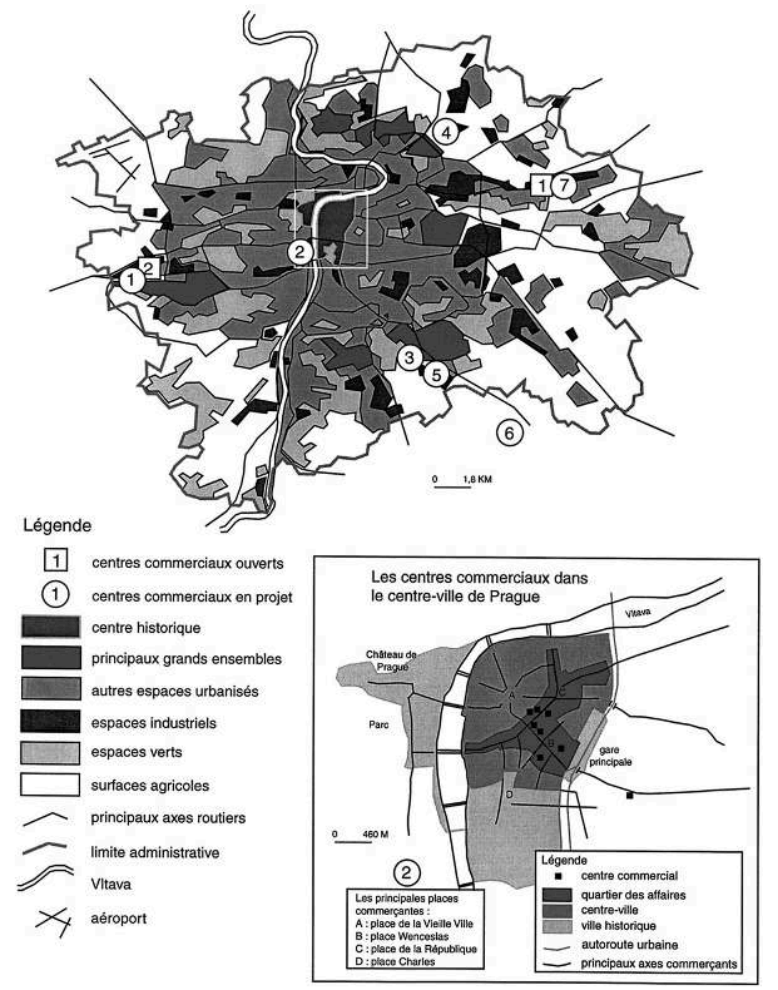

41 Le tableau 2 met en évidence l'importance des projets de centres commerciaux périphériques dont l'ouverture est prévue à la fin des années 1990 et au cours des premières années du XXIe siècle.

Tableau 2 : L'ouverture des centres commerciaux à Prague

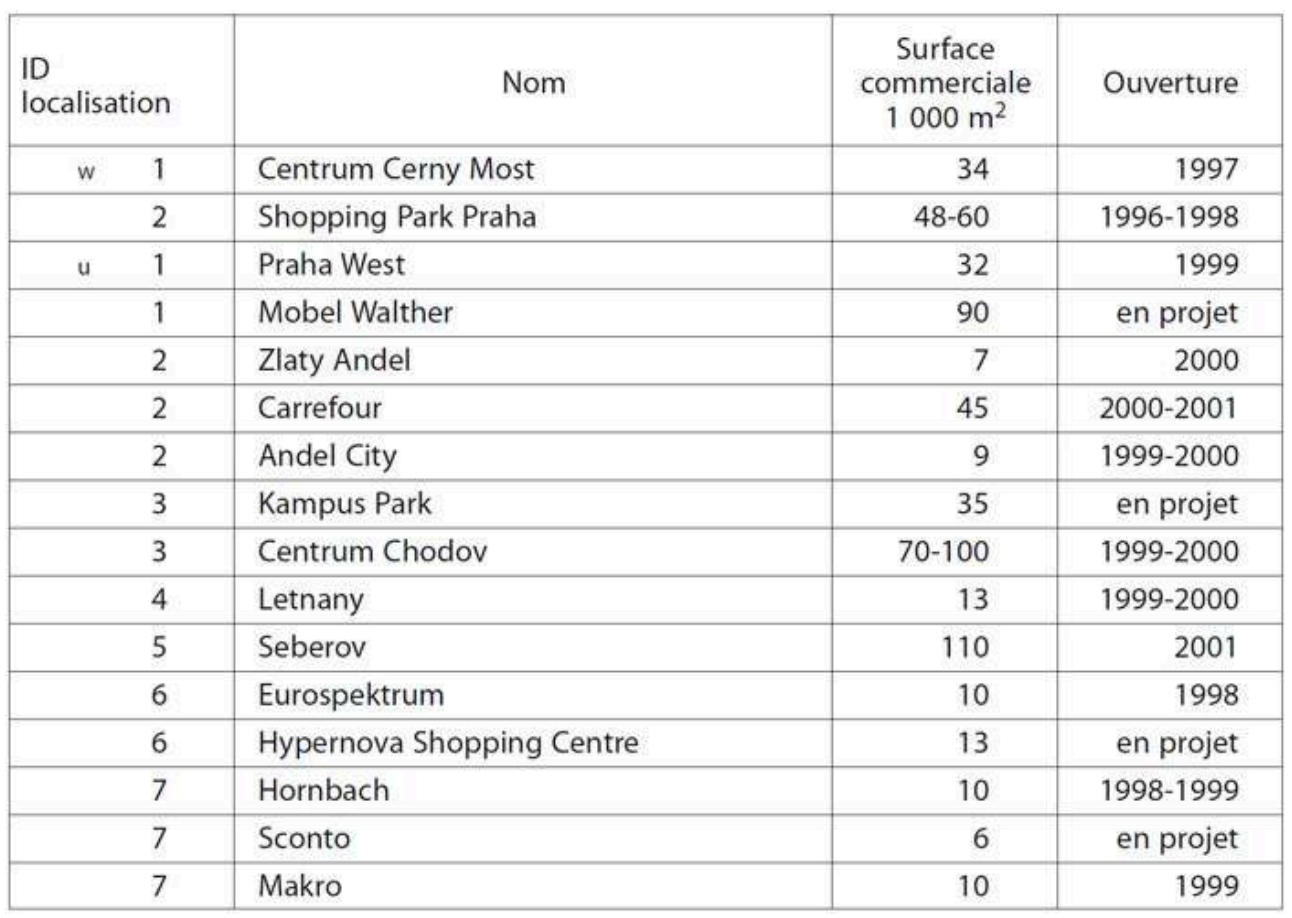

Sources : Haeley \& Baker, Prague, 1998 et Prague Business Journal, 12-18 janvier 1998 
Le tableau 3 permet de résumer les trois phases du développement du commerce de détail :

\section{Tableau 3 : le développement du commerce de détail à Prague}

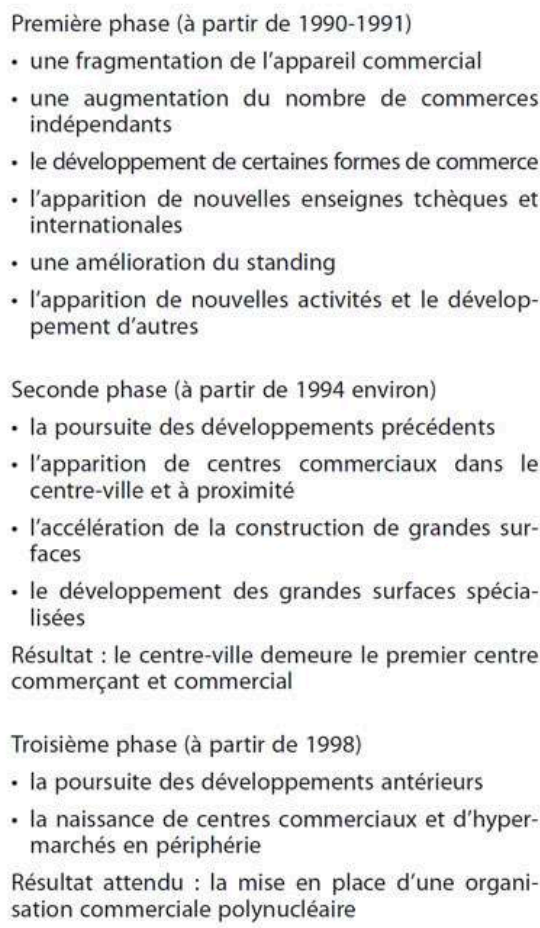

\section{Conclusion : l'importation du modèle d'organisation commerciale occidental}

On assiste ainsi à l'importation du modèle occidental d'organisation du commerce de détail et des services à caractère commercial sous l'effet de deux mécanismes :

\section{- L'adoption désirée du modèle par les autorités de la ville}

Les réformes de la Perestroïka et la chute des régimes communistes ont fait prendre conscience aux anciens pays du CAEM de l'échec de l'économie et de la politique socialistes et de leur retard par rapport aux pays d'Europe Occidentale. Cette prise de conscience d'un retard se retrouve également au niveau des villes. À Prague, les responsables de la ville tournent leur regard vers l'Ouest. Il s'agit, pour eux, d'adopter le modèle d'organisation commerciale des villes occidentales, tout en évitant les erreurs, en construisant des centres commerciaux périphériques qui doivent constituer des centres régionaux et des centres de quartier intermédiaires entre les précédents et le centre-ville. 


\section{- L'importation du modèle par les investisseurs occidentaux}

L'importation de ce modèle polynucléaire est également facilitée par la venue des investisseurs occidentaux. Pour une bonne représentativité, les grandes marques internationales implantent en premier lieu des points de vente dans le centre-ville. Dans leur optique, le centre de la capitale tchèque constitue un excellent tremplin vers les marchés de l'Est. Ceci ne signifie pas absence ailleurs. Elles se font plus discrètes dans les autres quartiers de la ville. Concernant les grandes surfaces commerciales, tous les projets sont le fait de sociétés internationales.

En conclusion, Prague connaît actuellement une évolution rapide de son organisation commerciale, comparable en partie au développement du commerce de détail occidental des années 1960 et 1970. Si les résultats finaux attendus sont les mêmes qu'en Europe Occidentale, les processus diffèrent.

\section{BIBLIOGRAPHIE}

Seuls les ouvrages précédés d'un astérisque sont cités dans le texte.

Baumann D. (1996). - La distribution des biens de consommation en République Tchèque, Direction des relations économiques extérieures, Les notes des postes d'expansion économique, ministère de l'Économie et des finances, Paris, août 1996, 67 p.

Blaha J. (1987). - « Tchécoslovaquie 1986-1990 : à petits pas », Le Courrier des Pays de l'Est, $\mathrm{n}^{\circ}$ 316, mars 1987, pp 54-59.

*Blaha J. (1989). - « Vers un marché noir unique à l'Est, l'essor du tourisme de shopping », Le Courrier des Pays de l'Est, $\mathrm{n}^{\circ}$ 342, août-septembre 1989, pp 40-48.

Blaha J. (1990). - «L'économie tchécoslovaque en 1989-1990 : la marche vers l'Ouest », Le Courrier des Pays de l'Est, n 349, avril 1990, pp 75-83.

CFCE (1997). - Un marché, République Tchèque, les éditions du CFCE, janvier 1997, 198 p.

Chaline C. (1980). - La dynamique urbaine, A.Colin, coll. U2, 254 p.

Earle J.S. et al. (1994). - Small privatization; the transformation of retail trade and consumer services in the Czech Republic, Hungary and Poland. Central European University Press. 1994. 301 p.

*Foucher M. (1993). - « Fragments d'Europe », The Prague Business Journal, Haeley \& Baker, Prague, Bureau de développement de la Ville de Prague, 12-18 janvier 1998.

*Gabrisch H. (1989). - « Réformes et politiques des prix à l'Est », Le Courrier des Pays de l'Est, $\mathrm{n}^{\circ}$ 342, août-septembre 1989, pp. 49-58.

*Kaplan J., Nosarzewska K. (1997). - Prague, Könemann Verlagsgesellschaft mbH, 360 p.

Plicka I. (1997). - Prague 97 metropolitan area report urban development of the city, City Development Authority Prague, 75 p. 
Pommois C. (1995). - Dynamique urbaine dans l'agglomération de Prague, la mutation du centre-ville et la transition, mémoire de maîtrise, Université Louis Pasteur, Strasbourg, 146 p.

RADIO FREE EUROPE RESEARCH (1987). - Czechoslovak SR/2, 06/02/87, CEDUCEE, La

Documentation Française, Paris, 2 p.

Schultz J. (1997). — « Mutations des structures et espaces urbains : les capitales de l'Europe médiane ». Dans : Maurel M.C. Recomposition de l'Europe médiane, SEDES, 1997, pp. 117-156.

Sedlar L. (1997). - « Prague’s retail revolution », The Prague Tribune, n²-february/unor 1997, pp. 10-17.

Statisticka Rocenka, 1979, 1981, 1984, 1986, 1991, Cesky Statisticky Urad.

*SUMMARY OF WORLD BROADCAST (1987). - Czechoslovak results in 1986, Report by federal Statistical Office, EE/8486/C/14, 07/02/87, CEDUCEE, La Documentation Française, Paris, 3 p.

Sykora L. (1997). - «Prague : mutations économiques et sociales ». Le Courrier des Pays de l'Est, $\mathrm{n}^{\circ} 416$, janvier-février 1997, pp. 24-39.

Sykora L, Simonickova I. (1994). - « From totalitarian urban managerialism to a liberalized real estate market : Prague's transformations in the early 1990s ». In : Barlow M., Dostal P., Hampl M., Development and Administration of Prague, Amsterdam, 1994, pp. 47-72.

Thailing S. (1994). - « Retail developments and legal changes ». In : The Prague Post, May 18 1994, pp. 15-16.

\section{NOTES}

1. Le parti communiste tchécoslovaque s'inquiéta de l'arrivée au pouvoir de Mikhaïl Gorbatchev en URSS en 1985. En 1986, une loi autorisa l'entreprise privée en Union Soviétique et une seconde loi, votée en 1987, autorisa l'autonomie des entreprises d'Etat. M. Gorbatchev venait de lancer la Perestroïka (réorganisation) et la Glasnost (transparence) qui allaient conduire à la chute des régimes communistes d'Europe Centrale et Orientale. En Tchécoslovaquie, Gustav Husak, qui dirigeait le pays depuis le Printemps de Prague en 1968, tenta de freiner l'évolution. Les dirigeants tchécoslovaques ne se prononcèrent en faveur de la réforme appelée « reconstruction complexe des mécanismes économiques » que sous la pression de l'Union Soviétique en 1987. (H. Gabrisch, 1989)

2. La partition de la Tchécoslovaquie, nommée «Divorce de Velours », n'eut lieu qu'au premier janvier 1993.

3. La privatisation des entreprises publiques devait se dérouler en deux grandes étapes. La petite privatisation consistait en une vente aux enchères des petites entreprises (commerces de détail, services et restaurants). Dans un premier temps, la participation étrangère fut interdite de ce processus. Les grandes unités furent privatisées par le biais de la grande privatisation au cours de laquelle les entreprises devaient élaborer un projet de privatisation. En 1993, le secteur de la distribution était entièrement privatisé.

4. Les immeubles qui n'ont pas pu être privatisés par le biais de la restitution, parce qu'il n'y a pas eu de déclaration de propriété ou parce qu'ils ont été construits après 1948, ont été soumis à la gestion des communes (Cf. note $n^{\circ}$ 6) de Prague. À partir de là, les autorités locales peuvent mettre les espaces communs des immeubles (en général, le rez-de-chaussée) en location pour des activités commerciales. 
5. Cette loi sur la protection des locataires qui exige le maintien des loyers à des niveaux très bas, ainsi que de trouver un logement équivalent en cas de vente ou de mise en location, représente un frein à la constitution d'un marché immobilier et à la restauration du bâti.

6. Les limites de la ville ont été définies de façon administrative. Des communes rurales et villages où s'étaient installés des personnes travaillant à Prague ont été successivement incorporées au territoire administratif de la ville en 1922, 1968 et 1974. Ces communes ont conservé leur maire et leur conseil municipal.

\section{RÉSUMÉS}

Suite à la chute du mur de Berlin et des régimes communistes en 1989, les pays d'Europe Centrale et Orientale instaurèrent une économie de transition devant faciliter le passage d'une économie dite de commandement à une véritable économie de marché. Des processus de restitution, de privatisation et de libéralisation furent amorcés à des vitesses plus ou moins rapides selon les pays. Dans ce nouveau contexte de transition, les villes d'Europe Centrale et Orientale connaissent des transformations qui touchent autant les paysages urbains que les organisations spatiales. À Prague, si le centre-ville est conforté dans sa place de premier centre commercial et commerçant de la ville, l'évolution actuelle se caractérise par la construction planifiée de véritables centres commerciaux près des zones résidentielles construites sous le régime communiste.

Since the fall of the Berlin Wall and the communist regimes in 1989, central and eastern European countries established an economy of transition to facilitate the change from a planned economy to a truly market economy. Processes of restitution, privatization and liberalization were engaged with varying rapidity according to the country. Under this new political and economic climate, the cities of Central and Eastern Europe are bringing change to urban landscapes as well as their spatial organisation. In Prague, if the center is confirmed in its role of the first central place of the city, the current development is the planned construction of new commercial centres near the residential areas built under the communist regime.

Nach dem Fall der Mauer in Berlin und der kommunistischen Regime 1989 richteten die Länder Mittel- und Osteuropas eine Übergangswirtschaft ein, um den Wechsel von der Planwirtschaft zu einer echten Marktwirtschaft zu erleichtern. Die Prozesse der Rückgabe, Privatisierung und Liberalisierung wurden je nach Land mehr oder weniger rasch eingeleitet. In diesem neuen Kontext des Übergangs erfuhren die Städte Mittel- und Osteuropas Veränderungen, die sowohl die Städtischen Landschaften wie die Raumorganisationen berührten. Wenn in Prag das Statzentrum verstärkt den Platz des ersten Handels- und Geschäftszentrums der Stadt einnimmt, wird die aktuelle Entwicklung charakterisiert durch die planmässige Errichtung echter Geschäftszentren nahe der unter dem kommunistischen Regime erbauten Wohnzonen. 
INDEX

Mots-clés : commerce de détail, dynamique urbaine, Europe Centrale, Prague

Keywords : Central Europe, Prague, retail, urban dynamic

Schlüsselwörter : Einzelhandel, Mitteleuropa, Prag, Stadtdynamik

\section{AUTEUR}

\section{CAROLE POMMOIS}

Laboratoire «Image et Ville » - UPRES-A 7011 CNRS - Université Louis-Pasteur - 3, rue de l'Argonne 67000 Strasbourg 